BMJ Paediatrics Open

\section{Impact of hypoglycaemia on neurodevelopmental outcomes in hypoxic ischaemic encephalopathy: a retrospective cohort study}

To cite: Tan JKG, Minutillo C, McMichael J, et al. Impact of hypoglycaemia on neurodevelopmental outcomes in hypoxic ischaemic encephalopathy: a retrospective cohort study. BMJ Paediatrics Open 2017;1:e000175. doi:10.1136/ bmjpo-2017-000175

Received 14 July 2017 Revised 16 August 2017 Accepted 17 August 2017
CrossMark

${ }^{1}$ Neonatal Intensive Care Unit, Princess Margaret Hospital for Children, Perth, Western Australia, Australia

${ }^{2}$ Centre for Neonatal Research and Education, The University of Western Australia, Perth, Western Australia, Australia ${ }^{3}$ State Child Development Centre, Perth, Western Australia Australia

Correspondence to Dr Jason Khay Ghim Tan; Jason. Tan@health.wa.gov.au

\section{ABSTRACT \\ Background Low blood glucose levels (BGLs) in infants are known to adversely affect neurodevelopmental outcomes. However, this risk is not well explored in infants with hypoxic ischaemic encephalopathy (HIE) that receive therapeutic hypothermia (TH). Additionally, little information is available on the optimal BGLs to target in infants with HIE. \\ Aim To explore the association between hypoglycaemia and neurodevelopmental outcomes at different BGL thresholds (2.6 and $3.0 \mathrm{mmol} / \mathrm{L}$ ) in neonates with HIE treated with $\mathrm{TH}$.}

Methods Retrospective cohort study. Clinical information and 2-year neurodevelopmental data using Bayley Scales of Infant Development, third edition (BSID-III) and disabilities were recorded for infants born in Western Australia with HIE and treated with TH between February 2008 and February 2012. Multivariable logistic regression models explored the association between hypoglycaemia and neurodevelopmental outcomes.

Results 122 infants underwent a total of 1616 BGL estimations before and during 72 hours of TH. Hypoglycaemia (BGL<2.6 mmol/L) occurred in $38 / 122(31 \%)$ infants and 11/122 (9\%) had recurrent hypoglycaemia (three or more episodes). Infants with recurrent hypoglycaemia $(<2.6 \mathrm{mmol} / \mathrm{L})$ had significantly lower mean BSID-III cognitive, language and socioemotional subscale scores. On multivariable analysis, recurrent hypoglycaemia $(<2.6 \mathrm{mmol} / \mathrm{L})$ was associated with increased odds of death or disability (adjusted OR 8.15; 95\% Cl 1.31 to 50.58; $p=0.024$ ). Recurrent hypoglycaemia $(<3.0 \mathrm{mmol} / \mathrm{L})$ during the first 12 hours of life was also associated with severe disability among survivors (adjusted OR 11.13; $95 \% \mathrm{Cl} 2.06$ to 59.89; $\mathrm{p}=0.005)$.

Conclusions Early recurrent hypoglycaemia was associated with increased risk of death or severe disability in neonates undergoing TH for HIE. Prospective studies are needed to identify the ideal target BGL in this population.

\section{INTRODUCTION}

Hypoxic ischaemic encephalopathy (HIE) in newborn infants is associated with high rates of mortality and neurodevelopmental morbidity. ${ }^{1-3}$ Therapeutic hypothermia
What is already known on this topic?

Infants with hypoxic ischaemic encephalopathy (HIE) are at high risk of neurodevelopmental disability.

$>$ Hypoglycaemia in HIE may increase the risk of unfavourable neurodevelopmental outcomes.

- This risk is not well explored in infants who receive therapeutic hypothermia.

\section{What this study hopes to add?}

- Hypoglycaemia is common in patients with HIE.

- Avoiding recurrent hypoglycaemia in infants with HIE is important to reduce secondary brain injury.

- We hypothesise that targeting blood glucose levels $>3.0 \mathrm{mmol} / \mathrm{L}$ may reduce the risk of unfavourable outcomes in these infants.

(TH) is known to improve short-term and long-term outcomes and has become the standard of care for moderate to severe HIE. ${ }^{4-6}$ Glucose control is an important management goal in intensive care units with studies showing that hypoglycaemia is associated with poor neurodevelopmental outcomes. ${ }^{7-9}$ However, there is controversy over the definition of hypoglycaemia in neonates with HIE. ${ }^{10-12}$ There are only four studies that have explored the effect of hypoglycaemia on the outcomes of neonates with HIE or suspected asphyxia, ${ }^{13-16}$ of which two studies included neonates with HIE that received $\mathrm{TH}^{1416}$

Hence we aimed to further explore the relationship between hypoglycaemia and neurodevelopmental outcomes in neonates with HIE that received TH. Another aim was to examine whether targeting higher blood glucose levels (BGLs) may reduce the risk of adverse neurodevelopmental outcomes. 


\section{METHODOLOGY}

This was a population-based retrospective case-control study of all neonates with HIE and managed with TH in Western Australia (WA) between February 2008 and February 2012. During this period all infants with HIE that required TH in WA were managed in the neonatal intensive care units (NICU) of Princess Margaret Hospital for Children (PMH) or King Edward Memorial Hospital for Women (KEMH). Both units function as a single clinical care unit and follow the same management protocols. Neonates with HIE were started on a maintenance intravenous infusion of $10 \%$ dextrose at $50 \mathrm{~mL} /$ $\mathrm{kg} /$ day without enteral feeds. A BGL below $2.6 \mathrm{mmol} / \mathrm{L}$ was managed with an intravenous bolus of $2 \mathrm{~mL} / \mathrm{kg}$ of $10 \%$ dextrose and the maintenance infusion increased by $10-20 \mathrm{~mL} / \mathrm{kg}$ /day with a repeat BGL within $60 \mathrm{~min}$. If the total fluid rate reaches $80-90 \mathrm{~mL} / \mathrm{kg} /$ day then the concentration was incrementally increased by $5 \%$. Hyperglycaemia was managed by reducing glucose infusion rates; insulin was not administered to any infant in this study. The majority of neonates were outborn and transferred to PMH or KEMH by the Newborn Emergency Transport Service of WA, which is based within the PMH neonatal unit and advise the same protocol as above when calls for transfer are received.

The cohort was identified using the neonatal database at both NICUs. All infants who were $\geq 35$ weeks postmenstrual age with HIE and treated with whole body $\mathrm{TH}$ were included in this study. TH was commenced in infants $\geq 35$ weeks postmenstrual age that were less than 6 hours old, had moderate or severe encephalopathy based on the Sarnat Grading Scale ${ }^{17}$ and had evidence of asphyxia as defined by the presence of at least two of the following criteria: (1) Apgar score $<6$ at $10 \mathrm{~min}$ or continued need for resuscitation with positive pressure ventilation or chest compressions at $10 \mathrm{~min}$, (2) any acute perinatal event that may result in HIE (ie, placental abruption, cord accident, prolonged fetal bradycardia, etc), (3) cord blood (or if cord blood is not available, arterial blood sample within $60 \mathrm{~min}$ of birth) with a $\mathrm{pH}<7.0 \mathrm{mmol} / \mathrm{L}$ or base deficit of 12 or more. Some infants with mild HIE received TH because of initial uncertainty of the severity of the encephalopathy prior to or during transfer. Some of these infants were subsequently reclassified to Sarnat grade 1 encephalopathy by the clinical team but completed 72 hours of cooling. These infants were included in the final analysis.

Infants were excluded if they had an inborn error of metabolism or congenital structural brain defect. Our unit protocol was to measure BGLs at least four hourly in the first 24 hours and then six hourly until the end of TH. More frequent measurements were undertaken in infants with poor glucose control.

The demographic, BGLs and 24-month neurodevelopmental outcome data were recorded. All BGLs from birth until the end of the 72 hours cooling period were recorded. Hypoglycaemia was defined as
BGL $<2.6 \mathrm{mmol} / \mathrm{L},{ }^{10}$ and hyperglycaemia was defined as $\mathrm{BGL}>8.3 \mathrm{mmol} / \mathrm{L} .{ }^{16}$ If more than one episode of hypoglycaemia or hyperglycaemia was detected within the same hour it was considered as one episode with the lower level recorded. BGLs were analysed using a point of care blood gas analyser at each NICU (ABL800 Flex; Radiometer, Copenhagen, Denmark). Glucose levels at the referring hospitals and during transfer were measured using a variety of methods, including reagent strips or iSTAT (Abbott Point of Care, Princeton, New Jersey, USA) and these were included in this analysis.

Neurodevelopmental measures recorded at 24 months include Bayley Scale of Infant and Toddler Development III (BSID-III) subscales (cognitive, language, motor and socioemotional), blindness, sensorineural deafness and cerebral palsy using the Gross Motor Function Classification System (GMFCS). Severe disability was defined as one or more of the following:

1. A cognitive or language subscale score $<70$ (standardised score of $100 \pm 15$, mean \pm SD) on BSID-III. Infants who were unable to complete an assessment due to their disabilities were given a score of 40 (4 SDs from the mean) for all subscales. ${ }^{18} 19$

2. Blindness: defined as $6 / 60$ vision in the better eye.

3. Sensorineural deafness: defined as the need to use bilateral hearing aids.

4. Cerebral palsy: GMFCS $\geq 3$.

Statistical analysis was done using IBM SPSS statistical software for Macintosh;V.21.0 (IBM, 2012; IBM, Armonk, New York, USA). Summary statistics are presented as median and IQR for skewed data and mean and SD for normally distributed continuous data. Frequency distributions and percentages were used to describe categorical data.

Infants were divided into two groups: no hypoglycaemia (all BGLs were $\geq 2.6 \mathrm{mmol} / \mathrm{L}$ ) and hypoglycaemic group (at least one BGL $<2.6 \mathrm{mmol} / \mathrm{L}$ ). The hypoglycaemic group was further divided into those with one episode, two episodes and three or more episodes of hypoglycaemia (recurrent). For univariate analysis, continuous normally distributed outcomes were

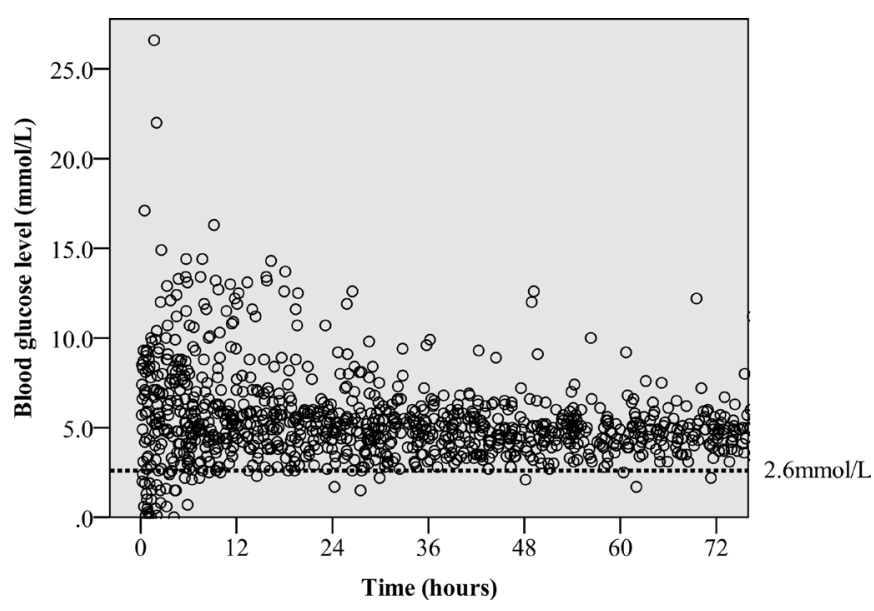

Figure 1 Variation of blood glucose levels over time. 
Table 1 Comparison of no hypoglycaemia versus hypoglycaemic infants

\begin{tabular}{|c|c|c|c|}
\hline Parameter & $\begin{array}{l}\text { No hypoglycaemia, } \\
\mathrm{n}=84\end{array}$ & $\begin{array}{l}\text { Hypoglycaemia group, } \\
n=38\end{array}$ & p Value \\
\hline Gestational age (mean, SD) & $39.2(1.9)$ & $39.0(1.9)$ & 0.609 \\
\hline Female (n, \%) & $38(45)$ & $14(37)$ & 0.433 \\
\hline Apgar score at $1 \mathrm{~min}$ (median, IQR) & $1(1-3)$ & $2(0.75-3)$ & $0.584^{*}$ \\
\hline Apgar score at $5 \mathrm{~min}$ (median, IQR) & $4(2-5)$ & $4(3-6)$ & $0.359^{\star}$ \\
\hline Gestational diabetes (n, \%) & $6(7)$ & $4(11)$ & 0.462 \\
\hline Cord pH (mean, SD) & $7.0(0.2)$ & $7.0(0.2)$ & 0.517 \\
\hline Cord lactate (mean, SD) & $11.0(4.6)$ & $14.2(5.3)$ & 0.007 \\
\hline Cord base excess (mean, SD) & $-13.7(6.8)$ & $-13.5(7.3)$ & 0.902 \\
\hline HIE stage on admission ( $\mathrm{n}, \%)$ & $\begin{array}{l}\text { Stage } 1-24(29) \\
\text { Stage 2-40 (48) } \\
\text { Stage 3-20 (24) }\end{array}$ & $\begin{array}{r}\text { Stage 1-6 (16) } \\
\text { Stage 2-23 (60) } \\
\text { Stage 3-9 (24) }\end{array}$ & $0.351^{*}$ \\
\hline Birth weight z-scores (mean, SD) & $-0.1(1.3)$ & $-0.1(1.4)$ & 0.934 \\
\hline Age at commencing hypothermia (min) (mean, SD) & $114.0(159.5)$ & $94.4(188.6)$ & 0.570 \\
\hline $\begin{array}{l}\text { Cardiopulmonary } \\
\text { resuscitation (n, \%) }\end{array}$ & $50(60)$ & $19(50)$ & 0.333 \\
\hline Resuscitation with epinephrine (n, \%) & $25(30)$ & $8(21)$ & 0.746 \\
\hline Withdrawal of care (n, \%) & $19(12)$ & $3(8)$ & 0.753 \\
\hline
\end{tabular}

*Mann-Whitney U test.

$\mathrm{HIE}$, hypoxic ischaemic encephalopathy.

compared using two-sided Student's t-test and MannWhitney U was performed for non-normally distributed continuous outcomes. Categorical outcomes were compared using Fisher's exact test. Logistic regression models were used to analyse the relationship between neurodevelopmental outcomes and hypoglycaemia as defined at two different levels $(2.6$ and $3.0 \mathrm{mmol} / \mathrm{L})$ and time periods (hypoglycaemic episodes in the first 12 hours of life and first 72 hours of life). Effect size estimates were derived after adjusting for HIE stage, time of commencement of TH and any episode of hyperglycaemia. Statistical significance was defined as a $p$ value of less than 0.05 .

Hospital ethics and governance committee approval was obtained for this study. Parent consent was deemed not necessary given the retrospective nature of the study.

\section{RESULTS}

A total of 122 infants were included in this analysis of which $18(14.7 \%)$ died in the first 2 years of life (all had HIE Sarnat stage 3). Eight died during the first 72 hours of life, eight between 72 hours and 28 days and the remaining two at 17 and 22 months of age. Of the 104 survivors, follow-up data were available for $87(84 \%)$ infants. Severe disability was seen in 15/87 (17\%) infants. Nine had cerebral palsy, four were deaf, one was blind and 11 had a BSID-III cognitive or language score $<70$ ). None of the infants reclassified to Sarnat grade 1 died or had severe disability.

\section{Glucose homoeostasis}

A total of 1616 BGL measurements were recorded from 122 infants from birth to the end of TH or death when this occurred within 72 hours of birth. Half of the samples were measured in the first 24 hours of life. Of the 122 infants, $38(31 \%)$ had at least one episode of hypoglycaemia $(\mathrm{BGL}<2.6 \mathrm{mmol} / \mathrm{L}) ; 22(18 \%)$ had two episodes and $11(9 \%)$ had three or more episodes of hypoglycaemia. Glucose homoeostasis of the infants appeared to improve over the period of $\mathrm{TH}$ as seen on the scatterplot (figure 1). The majority $(86.5 \%)$ of hypoglycaemic episodes occurred during the first 24 hours, $9.5 \%$ in the second 24 hours and $4 \%$ in the last 24 hours of TH.

Table 1 compares the basic demographic data of the no hypoglycaemia group and hypoglycaemic group. The only significant difference was that the hypoglycaemic group had a higher cord blood lactate level.

\section{Relationship between hypoglycaemia and neurodevelopmental outcomes}

The BSID-III and adverse neurodevelopmental outcomes at 24 months of life are shown in table 2, which compares infants with no episodes of hypoglycaemia (baseline) to infants who have varying frequency of hypoglycaemia. The cognitive, language and motor mean BSID-III subscale scores were significantly lower in the group with three or more episodes of hypoglycaemia than the no hypoglycaemia group. Deafness, any severe disability and composite of death and/or severe disability were significantly more common in the group with three or more episodes of 
Table 2 Neurodevelopmental outcomes at 24 months

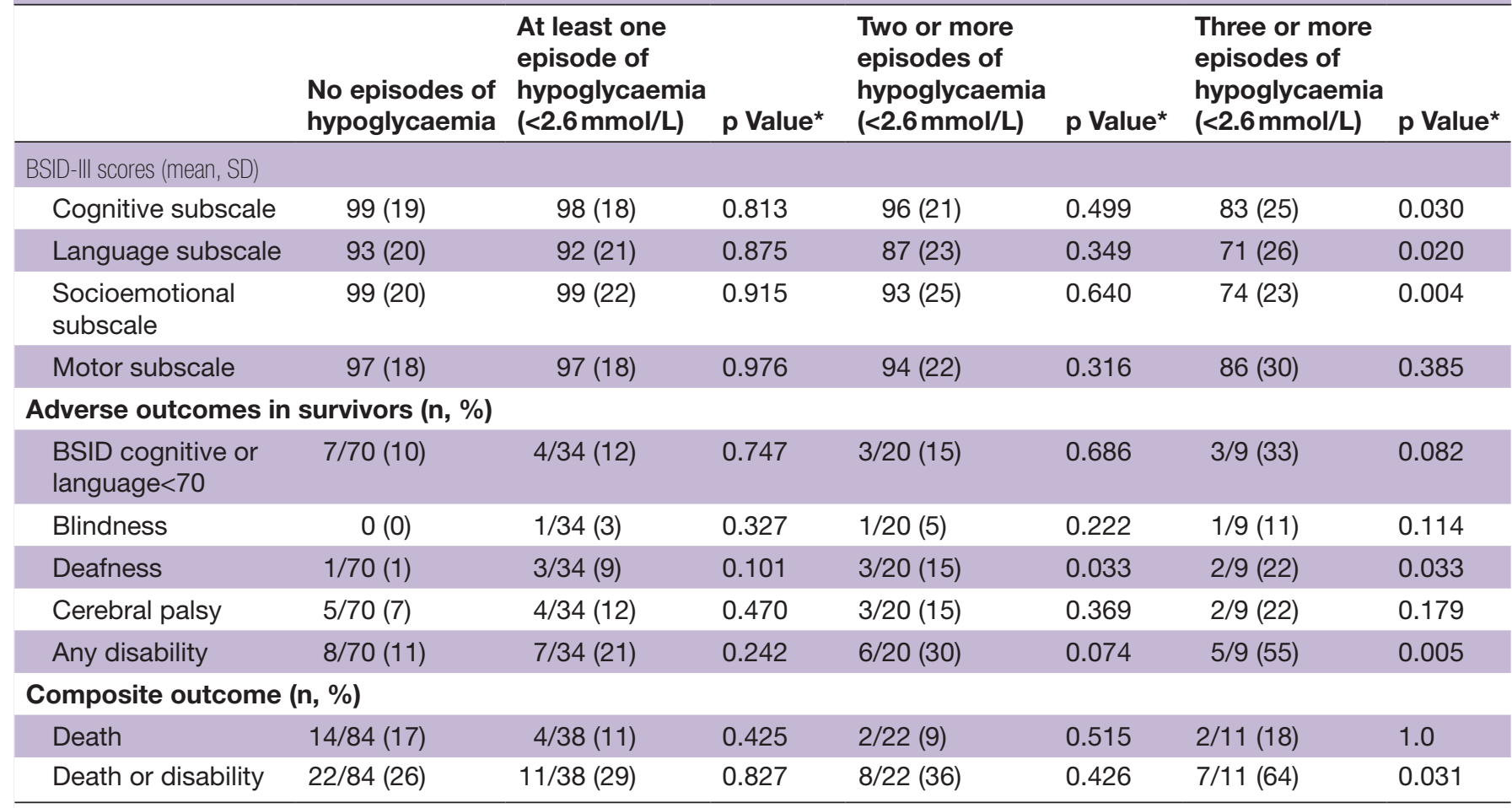

*p Value compared with no hypoglycaemia group.

BSID-III, Bayley Scale of Infant and Toddler Development III.

hypoglycaemia compared with the no hypoglycaemia group. Mortality rate was similar between all the hypoglycaemic groups and the no hypoglycaemia group.

On univariate analysis, hyperglycaemia was significantly associated with severe disability and the composite of death and/or severe disability; however, this association was lost on multivariable analysis.

After adjusting for HIE stage, hyperglycaemia and time of starting $\mathrm{TH}$, infants with three or more episodes of hypoglycaemia (BGL $<2.6 \mathrm{mmol} / \mathrm{L}$ ) had significantly higher odds of severe disability alone (table 3 ) or the composite of death and/or severe disability than infants with nil episodes (table 4). Hypoglycaemia within the first 12 hours of life worsens the odds for severe disability and the composite of death and/or severe disability.

When using a BGL threshold of $3.0 \mathrm{mmol} / \mathrm{L}$, hypoglycaemia in the first 72 hours of life was not associated with severe disability alone (table 3) or the composite of death and/or severe disability (table 4). However, infants with three or more episodes of hypoglycaemia in the first 12 hours of life had significantly higher odds of severe disability alone and the composite of death and/or severe disability than infants with nil episodes. This association was still significant in this subset after adjusting for hyperglycaemia and time of starting $\mathrm{TH}$.

\section{DISCUSSION}

This study highlights the fact that glucose homoeostasis is poor in the first 72 hours of life for infants suffering from
HIE, which predisposes them to episodes of hypoglycaemia. The results also suggest that recurrent episodes of hypoglycaemia (BGL $<2.6 \mathrm{mmol} / \mathrm{L}$ ) are associated with worse neurodevelopmental outcomes.

There is no universal agreement on the definition of neonatal hypoglycaemia in otherwise healthy neonates, although maintaining levels above $2.5 \mathrm{mmol} / \mathrm{L}$, especially after the first 24 hours of life, is recommended. ${ }^{10}$ A large proportion of our study group $(31 \%)$ had at least one episode of hypoglycaemia (BGL $<2.6 \mathrm{mmol} / \mathrm{L}$ ) with almost $10 \%$ having three or more episodes of hypoglycaemia. The prevalence of any episode of hypoglycaemia in our cohort was similar to studies that involved $\mathrm{TH}$ in newborns, with rates ranging from $12 \%$ to $28 \% .{ }^{1620}$ Similar to other studies, the glucose homoeostasis improved over the first 72 hours of life with the majority of BGLs falling between 3 and $8.3 \mathrm{mmol} / \mathrm{L}$ after the first 48 hours of life. ${ }^{1516}$

We found that recurrent hypoglycaemia, particularly in the first 12 hours of life, was associated with adverse neurodevelopmental outcomes after adjusting for HIE stage, time of commencement of $\mathrm{TH}$ and any episode of hyperglycaemia. Three studies have reported on the association of early hypoglycaemia with long-term neurodevelopmental outcomes in infants with HIE, ${ }^{14-16}$ Nadeem et $a l^{15}$ found that early hypoglycaemia was associated with worse neurodevelopmental outcome at 24 months of age in infants with HIE, however, when adjusted for HIE stage this was no longer significant; the difference between this and our study may be due to the larger 


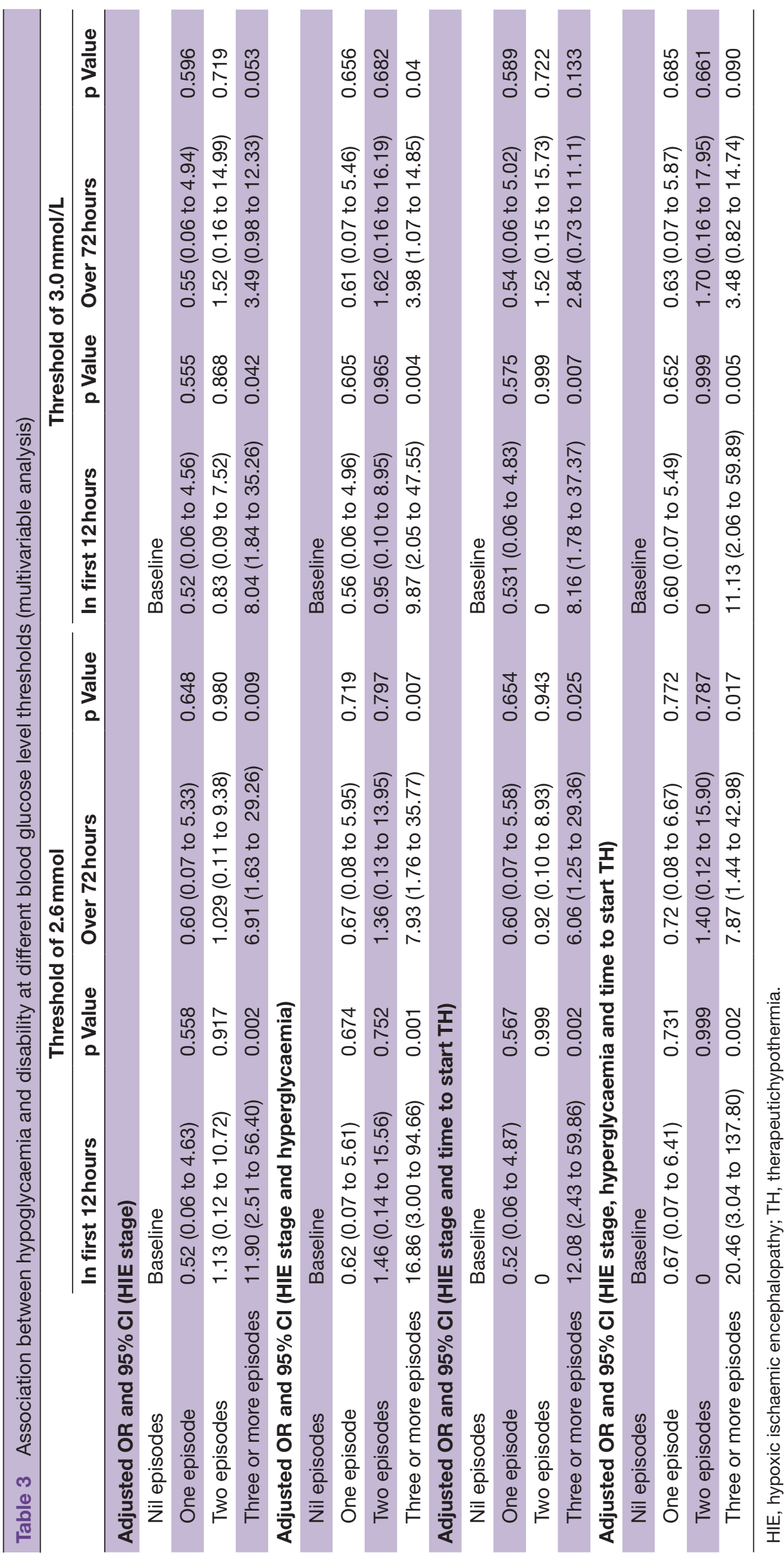




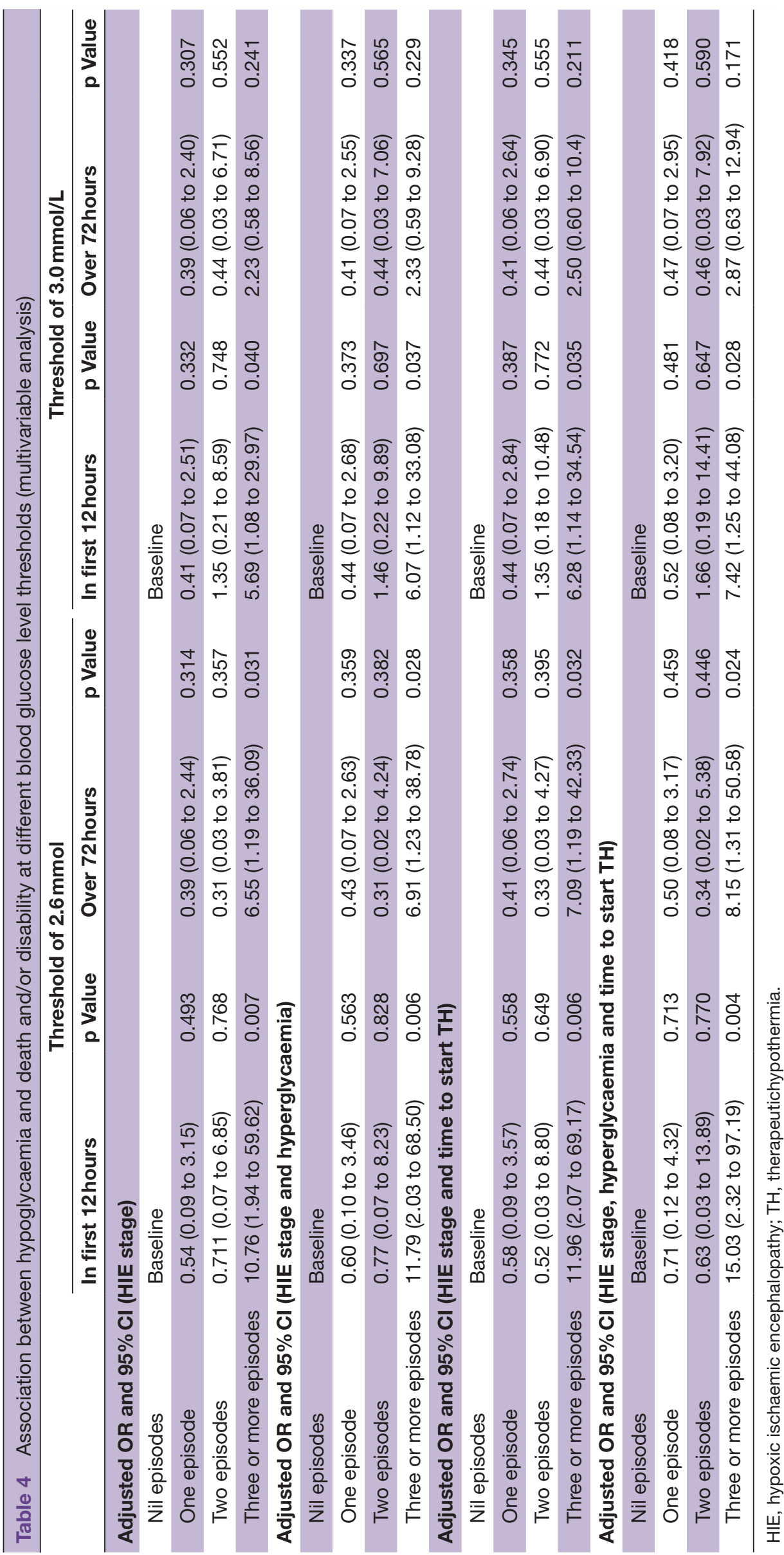


number of infants in our study, 52 vs 122 . Two studies, Basu et al and Tam et al, had infants who received TH, making up 50\% and $12 \%$ of their cohort, respectively. ${ }^{14} 16$ Tam $e t a l^{4}$ found that hypoglycaemia was a risk factor for future corticospinal tract injury and there was a 15-point lower cognitive and language score on the BSID-III scale. Basu $e a^{16}$ found that infants from the CoolCap trial that had hypoglycaemia were at significantly higher risk of an unfavourable neurodevelopmental outcome. These associations remained significant after adjusting for birth weight, Apgar score, $\mathrm{pH}$, Sarnat stage and hypothermia group.

An interesting finding of our study was that even when we used a cut-off of $3.0 \mathrm{mmol} / \mathrm{L}$ for the definition of hypoglycaemia, the association between recurrent hypoglycaemia in the first 12 hours of life and death and/or severe disability among survivors persisted. These findings may be due to the small numbers. There are no comparative studies conducted that support this finding. Hence, we can only hypothesise that targeting a threshold of $3.0 \mathrm{mmol} / \mathrm{L}$ in the first 12 hours of life instead of the traditional $2.6 \mathrm{mmol} / \mathrm{L}$ may result in additional benefits for these vulnerable infants. This requires further investigation.

The limitations of this study are its retrospective cohort design, small sample size and the heterogeneous population group (inborn vs outborn) and absence of data on Apgar scores at $10 \mathrm{~min}$ in many of the infants. Despite having a heterogeneous population the clinical characteristics of inborn and out-born groups were similar with regards to demographic data, stage of HIE, biochemical and clinical markers of severity and rates of hypoglycaemia. Although there was a $20 \%$ rate of loss to follow-up at 2 years, the study and lost to follow-up group were similar with regards to demographic data, stage of HIE, biochemical and clinical markers of severity and rates of hypoglycaemia. Another limitation was that the cut-off values that we chose to define hypoglycaemia for analysis were arbitrary.

Our study confirms that hypoglycaemia frequently complicates the care of infants born with HIE and that recurrent hypoglycaemia (BGL $<2.6 \mathrm{mmol} / \mathrm{L}$ ) in this setting is associated with significantly higher risk of death or severe disability. Larger prospective studies are required to establish the optimal BGLs in infants undergoing TH for HIE. Early anticipation and prompt management to prevent early, recurrent hypoglycaemia is essential to improve neurodevelopmental outcomes in infants with HIE receiving TH.

Contributors JKGT designed the study and data collection instruments, collected the data, carried out the initial analyses, drafted the initial manuscript, reviewed and approved the final manuscript as submitted. CM assisted in design of the study, reviewed and revised the manuscript and approved the final manuscript as submitted. JM assisted in design of the study, reviewed and revised the manuscript and approved the final manuscript as submitted. SR conceptualised the study and assisted in design of the study, reviewed and revised the manuscript and approved the final manuscript as submitted. All authors approved the final manuscript as submitted and agree to be accountable for all aspects of the work.

Competing interests None declared.

Ethics approval Princess Margaret Hospital Human Research Ethics Committee.

Provenance and peer review Not commissioned; externally peer reviewed.

Open Access This is an Open Access article distributed in accordance with the Creative Commons Attribution Non Commercial (CC BY-NC 4.0) license, which permits others to distribute, remix, adapt, build upon this work non-commercially, and license their derivative works on different terms, provided the original work is properly cited and the use is non-commercial. See: http://creativecommons.org/ licenses/by-nc/4.0/

(c) Article author(s) (or their employer(s) unless otherwise stated in the text of the article) 2017. All rights reserved. No commercial use is permitted unless otherwise expressly granted.

\section{REFERENCES}

1. Robertson CM, Perlman M. Follow-up of the term infant after hypoxic-ischemic encephalopathy. Paediatr Child Health 2006;11:278-82.

2. Levene MI, Sands C, Grindulis $H$, et al. Comparison of two methods of predicting outcome in perinatal asphyxia. Lancet 1986;1:67-9.

3. Volpe JJ. Neurology of the newborn. 5th edn. Philadelphia: Saunders/Elsevier, 2008.

4. Johnston MV, Fatemi A, Wilson MA, et al. Treatment advances in neonatal neuroprotection and neurointensive care. Lancet Neurol 2011;10:372-82.

5. Shah PS. Hypothermia: a systematic review and meta-analysis of clinical trials. Semin Fetal Neonatal Med 2010;15:238-46.

6. Edwards AD, Brocklehurst P, Gunn AJ, et al. Neurological outcomes at 18 months of age after moderate hypothermia for perinatal hypoxic ischaemic encephalopathy: synthesis and meta-analysis of trial data. BMJ 2010;340:c363.

7. Boardman JP, Wusthoff CJ, Cowan FM. Hypoglycaemia and neonatal brain injury. Arch Dis Child Educ Pract Ed 2013;98:2-6.

8. Burns CM, Rutherford MA, Boardman JP, et al. Patterns of cerebral injury and neurodevelopmental outcomes after symptomatic neonatal hypoglycemia. Pediatrics 2008;122:65-74.

9. Lucas A, Morley R, Cole TJ. Adverse neurodevelopmental outcome of moderate neonatal hypoglycaemia. BMJ 1988;297:1304-8.

10. Adamkin DH. Neonatal hypoglycemia. Semin Fetal Neonatal Med 2017;22:36-41.

11. Rozance PJ, Hay WW. Hypoglycemia in newborn infants: Features associated with adverse outcomes. Biol Neonate 2006;90:74-86.

12. Hay WW, Raju TN, Higgins RD, et al. Knowledge gaps and research needs for understanding and treating neonatal hypoglycemia: workshop report from Eunice Kennedy Shriver National Institute of Child Health and Human Development. J Pediatr 2009;155:612-7.

13. Salhab WA, Wyckoff MH, Laptook AR, et al. Initial hypoglycemia and neonatal brain injury in term infants with severe fetal acidemia. Pediatrics 2004;114:361-6.

14. Tam EW, Haeusslein LA, Bonifacio SL, et al. Hypoglycemia is associated with increased risk for brain injury and adverse neurodevelopmental outcome in neonates at risk for encephalopathy. J Pediatr 2012;161:88-93.

15. Nadeem M, Murray DM, Boylan GB, et al. Early blood glucose profile and neurodevelopmental outcome at two years in neonatal hypoxicischaemic encephalopathy. BMC Pediatr 2011;11:10.

16. Basu SK, Kaiser JR, Guffey D, et al. Hypoglycaemia and hyperglycaemia are associated with unfavourable outcome in infants with hypoxic ischaemic encephalopathy: a post hoc analysis of the CoolCap Study. Arch Dis Child Fetal Neonatal Ed 2016;101:F14 9-F155.

17. Sarnat HB, Sarnat MS. Neonatal encephalopathy following fetal distress. A clinical and electroencephalographic study. Arch Neurol 1976;33:696-705.

18. Doyle LW, Davis PG, Schmidt B, et al. Cognitive outcome at 24 months is more predictive than at 18 months for IQ at 8-9 years in extremely low birth weight children. Early Hum Dev 2012;88:95-8.

19. Doyle LW, Roberts G, Anderson PJ. Victorian Infant Collaborative Study G. Outcomes at age 2 years of infants. J Pediatr 2010;156:49-53.

20. Jacobs SE, Berg M, Hunt R, et al. Cooling for newborns with hypoxic ischaemic encephalopathy. Cochrane database of systematic reviews 2013;1:CD003311. 